\title{
BMJ Open Quality Improving a process to obtain hepatitis B serology among patients treated with infliximab at a large urban children's hospital
}

\author{
M Raphaelle Jean, ${ }^{1}$ Ann Weaver, ${ }^{1}$ Teresa Mastin-Diebold, ${ }^{1}$ Krista Kissinger, ${ }^{1}$ \\ Emily A Smitherman, ${ }^{2}$ Leslie Favier, ${ }^{2}$ Lara Danziger-Isakov, ${ }^{3}$ Elizabeth Williams, ${ }^{1}$ \\ Rebecca C Brady, ${ }^{3}$ Jennifer Huggins, ${ }^{2}$ Lee A Denson, ${ }^{1}$ Shehzad A Saeed, ${ }^{1}$ \\ Pamela Morgan, ${ }^{1}$ Dana Michelle Hines Dykes ${ }^{1}$
}

To cite: Jean MR, Weaver A, Mastin-Diebold T, et al. Improving a process to obtain hepatitis B serology among patients treated with infliximab at a large urban children's hospital.BMJ Open Quality 2017;6:e000092. doi:10.1136/ bmjoq-2017-000092

Received 21 April 2017 Revised 5 September 2017 Accepted 23 September 2017

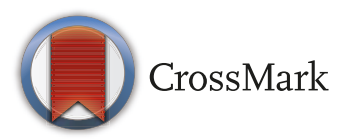

${ }^{1}$ Division of Pediatric Gastroenterology, Hepatology, and Nutrition, Department of Pediatrics, Cincinnati Children's Hospital Medical Center, Cincinnati, Ohio, USA

${ }^{2}$ Division of Rheumatology, Department of Pediatrics, Cincinnati Children's Hospital Medical Center, Cincinnati, Ohio, USA

${ }^{3}$ Division of Infectious Diseases, Department of Pediatrics, Cincinnati Children's Hospital Medical Center, Cincinnati, Ohio, USA

Correspondence to Dr Dana Michelle Hines Dykes; chellydykes@gmail.com

\section{ABSTRACT}

Background Hepatitis B infection is a significant public health challenge despite improvements in vaccination efforts. Patients such as those on chronic immunosuppressive therapy for inflammatory bowel disease (IBD) or rheumatic disease may incur greater risk. The risk of reactivation of hepatitis $B$ while on immunosuppressive therapy may have mortality rates up to $25 \%$. These patients should be screened for acute or chronic infection and vaccinated if necessary. Our aim was to reliably complete hepatitis $B$ screenings in patients receiving infliximab at Cincinnati Children's Hospital Medical Center (CCHMC).

Methods Eligible patients included all patients with gastroenterology (GI) IBD and rheumatology receiving infliximab between 0ctober 2015 and March 2016. Using quality improvement methodology and the 'plan-do-study-act' (PDSA) approach, interventions centred around education of clinical providers, previsit planning and the development of 'talking points' for patients.

Results An initial screen of the IBD population revealed that $48 \%$ of the IBD patient population had been screened for anti-HBs alone, but no patients from Gl or rheumatology divisions had a complete set of hepatitis $B$ serology prior to the intervention including anti-Hep B Core and Hep B Surface Antigen. Seven PDSA cycles were performed during the 32week intervention period, resulting in an increase in patients screened from $0 \%$ to $85 \%$. By March 2016, a total of 251 patients ( $201 \mathrm{Gl}, 50$ rheumatology) had up-to-date hepatitis B serology screening. Automated ordering of the hepatitis B serology and 'talking points' for the provider had the greatest impact on successful screening.

Conclusions We developed a method to obtain hepatitis B serology on at-risk patients on infliximab within two subspecialty divisions within a large children's hospital. Next steps will be to develop a process to reliably provide vaccines for patients who are seronegative, expand this process to all patients who are identified as immunocompromised within $\mathrm{Gl}$ and rheumatology and then expand this process to other divisions at the CCHMC.

\section{PROBLEM}

Current guidelines suggest monitoring for hepatitis B immunity and attempting to ensure adequate immunity for patients on chronic immunosuppressive therapy, ideally prior to the start of these therapies, with particular attention to those on biologics such as infliximab. ${ }^{1}$ In our gastroenterology (GI) inflammatory bowel disease (IBD) and rheumatology programme at Cincinnati Children's Hospital Medical Center (CCHMC), we have identified a gap in our ability to reliably assess for hepatitis B virus (HBV) immunity and to properly ensure that patients who are lacking appropriate immune response receive appropriate booster vaccinations. Our aim was to develop a process to reliably complete HBV screenings on patients receiving infliximab within the paediatric GI and rheumatology divisions at the CCHMC. A search in the electronic medical record shared by the hospital found that of the 598 patients with IBD at the CCHMC, only $48 \%$ ( $n=286$ ) have been screened for HBV immunity using anti-HBs alone (figure 1), and of these only $35 \% \quad(n=101)$ of the patients who were screened had documented immunity to HBV. Among our 62 rheumatology patients on infliximab, the majority of whom are diagnosed with juvenile idiopathic arthritis, there also was no set process to identify and screen them for hepatitis B immunity. None of these patients had a complete set of hepatitis B titres which also included Hep B Core antibody (anti-HBc) and Hep B Surface Antigen (HBsAg). We intended to use quality improvement methodology to improve hepatitis B screening and 


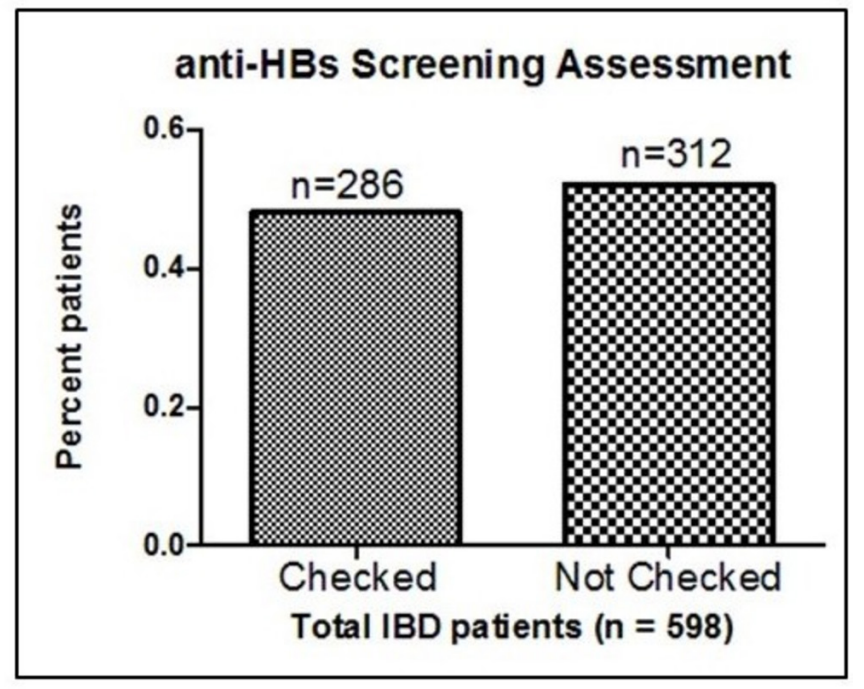

Figure 1 Anti-HB screening assessment among entire inflammatory bowel disease (IBD) patient population at the Cincinnati Children's Hospital Medical Center.

ultimately vaccination rates for this vulnerable population.

\section{BACKGROUND}

Autoimmune conditions requiring immune suppression are among the most common chronic diseases in paediatric gastroenterology and rheumatology practices. Conditions such as ulcerative colitis and Crohn's disease, collectively referred to as IBDs, as well as juvenile idiopathic arthritis are chronic inflammatory disorders encountered frequently in paediatrics. ${ }^{2}$ These diseases are debilitating conditions with unpredictable courses and challenging treatments. ${ }^{23}$ Although immunosuppressive medications have resulted in improved outcomes, these therapies may place patients at increased risk of complications from potentially preventable infections such as hepatitis $\mathrm{B} .{ }^{4-6}$

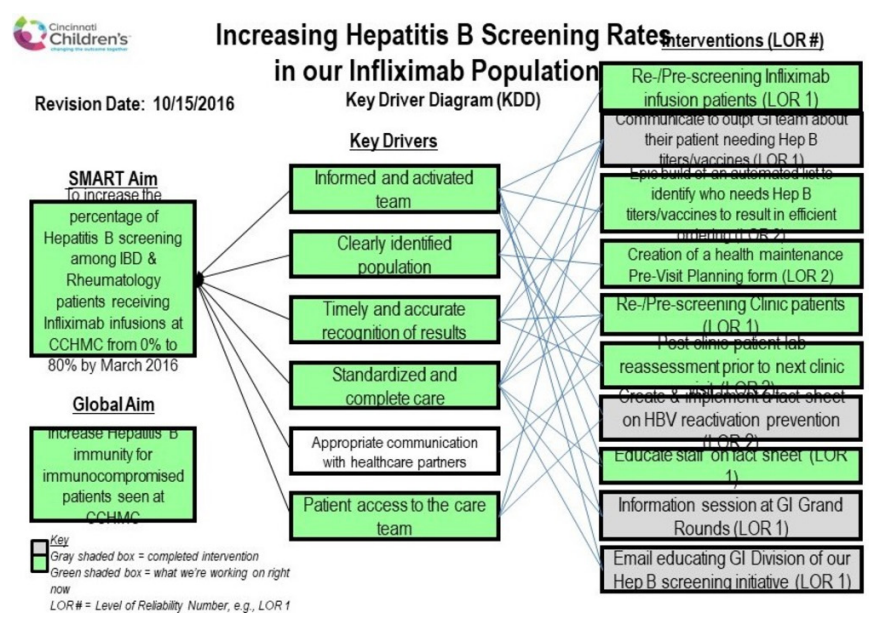

Figure 2 Key driver diagram for hepatitis B screening. CCHMC, Cincinnati Children's Hospital Medical Center; HBV, hepatitis B virus; IBD, inflammatory bowel disease.
Vaccination for hepatitis B is currently performed in infancy, but can be completed later in life if necessary. Currently both licensed single-antigen hepatitis $B$ vaccines administered intramuscularly produce a $>95 \%$ sero-protection rate in adolescents. ${ }^{5}$ During 1995-2005, incidence of acute hepatitis B in the USA among children and adolescents declined by $94 \%$, coincident with an increase in hepatitis $\mathrm{B}$ vaccination coverage. ${ }^{5}$ Immunocompetent persons, even those vaccinated during infancy, who achieve appropriate anti-HBs concentrations $(>10 \mathrm{mIU} / \mathrm{mL})$ after vaccination have virtually complete protection against both acute disease and chronic infection even if anti-HBs concentrations subsequently decline to $<10 \mathrm{mIU} / \mathrm{mL}^{.}{ }^{7-11}$ Some vaccinations such as the hepatitis $B$ vaccine may be less effective in patients with underlying immune conditions or who are already receiving immune suppressive therapy, in which a reduced persistence of immunological memory may be seen over time. ${ }^{6}{ }^{12}$ Although immunogenicity is lower among immunocompromised persons, those who achieve and maintain a protective antibody response before exposure to HBV have a high level of protection from infection. ${ }^{5}$

Despite the above improvements in vaccination efforts, hepatitis B infection remains a significant public health challenge. For patients receiving biological therapies such as anti-tumour necrosis factor alpha treatments, reactivation or newly acquired hepatitis $\mathrm{B}$ infection can be associated with an abrupt increase in HBV replication, jaundice, elevated serum aminotransferase levels and can pose a significant risk of morbidity and mortality in patients receiving these agents, with mortality rates reported as high as $25 \%{ }^{13-16}$ One cross-sectional singlecentre study evaluating 100 paediatric IBD patients on infliximab at a large, urban tertiary care facility demonstrated that a significant minority $(13 \%)$ of study participants had not been vaccinated against the HBV and an additional $44 \%$ of previously vaccinated patients lacked protective anti-HBs levels. ${ }^{4}$ Among these non-immune patients receiving HBV immunisation, only $14 \%$ of patients appeared at risk for HBV infection due to inability to elicit immunity to the virus. ${ }^{11}$ Given risk of severe disease if affected, vaccination screening and immunisation for patients without protective antibodies represents an opportunity for disease prevention in this vulnerable population even if they have already started infliximab.

\section{BASELINE MEASUREMENT}

Our first baseline measurement was performed using the larger IBD population rather than the smaller rheumatology patients and found that in a population of 598 patients $<50 \%$ (286) had been screened with anti-HBs alone (figure 1). We chose to narrow our scope to both rheumatology and gastroenterology patients receiving infliximab $(n=237)$ since these were viewed as the highest risk patients and were followed up most regularly and 


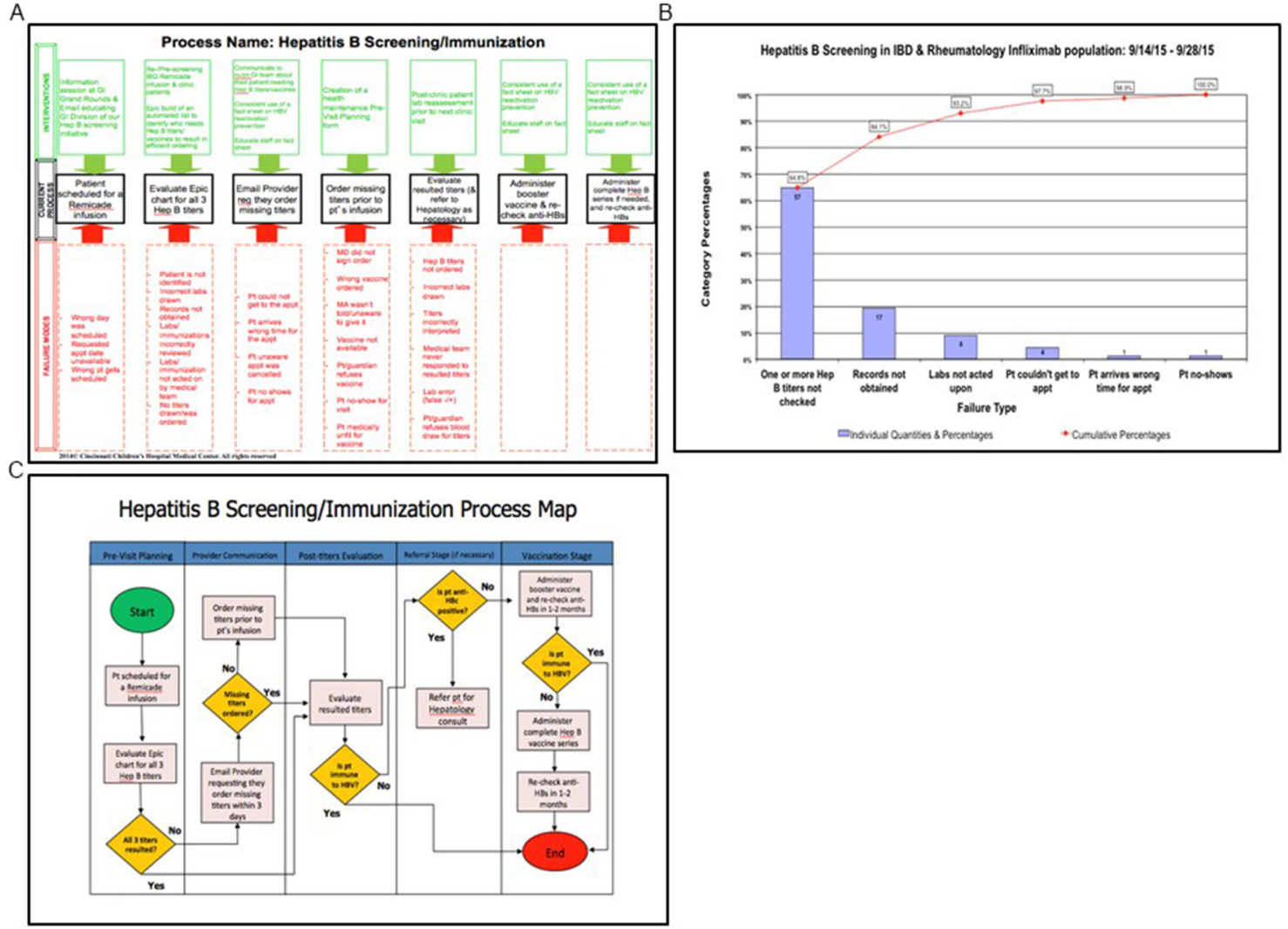

Figure 3 Analytic tools to design and measure improvements. (A) Failure mode analysis for hepatitis B screening. (B) Pareto chart demonstrating the most common hepatitis B screening process failures within the inflammatory bowel disease (IBD) and rheumatology population. (C) Hepatitis B screening/immunisation process map.

rapidly for reassessment (every $4-8$ weeks for infusions). Among the population receiving infliximab, we documented complete screening serology (including anti-HBc, anti-HBs and HBsAg) in $0 \%$ of patients at the onset of the project. Our team's specific, measurable, achievable, realistic, timely (SMART) aim was that by March 2016 we would increase the per cent of complete HBV screenings (all three titres) each week from $0 \%$ to $80 \%$ among patients with IBD and rheumatology receiving infliximab infusions at the CCHMC. Our global/long-term aim is to increase HBV immunity for immunocompromised patients seen at the CCHMC by improving vaccination rates for at-risk patients.

\section{DESIGN}

Prior to making changes to the current system, we identified key drivers that would optimise our system's ability to achieve and maintain a process for monitoring and improving hepatitis B screening and immunity (figure 2). These drivers included an informed and activated team, a clearly identified population, timely and accurate recognition of results, standardised and complete care, appropriate communication with healthcare partners and patient access to the care team.

Initial steps prior to designing interventions centred around a formal failure analysis (figure 3A), Pareto analysis (figure $3 \mathrm{~B}$ ) and process map of the hepatitis $\mathrm{B}$ screening process (figure 3C). Opportunities for improvement included education, appropriate and timely recognition of at-risk patients, and building standardisation and automation into the current processes.

The main interventions included re-screening/ pre-screening infliximab infusion patients, creation and implementation of a fact sheet on HBV reactivation prevention (which included a standardised algorithm), Epic build of an automated list to identify at-risk patients, and the creation and implementation of a health maintenance previsit planning (PVP) form to be used in our cohort of infliximab infusion patients and ultimately all clinic patients. Prior to beginning the above interventions, early plan-do-study-act (PDSAs) were completed using education/information sessions with GI/rheumatology medical providers to increase their knowledge regarding the improvement project goals and objectives. These sessions were repeated periodically throughout the 6-month timeline of the project and repeated or altered if gaps in medical providers' understanding of the project objectives were identified.

The backbone of the HBV screening process for our patients on infliximab therapy is founded in the integration of multiple PDSAs which worked well as a bundle (education, automated list, previsit order entry) and has led to the development of an automated system to be incorporated into routine clinical practice through PVP. 


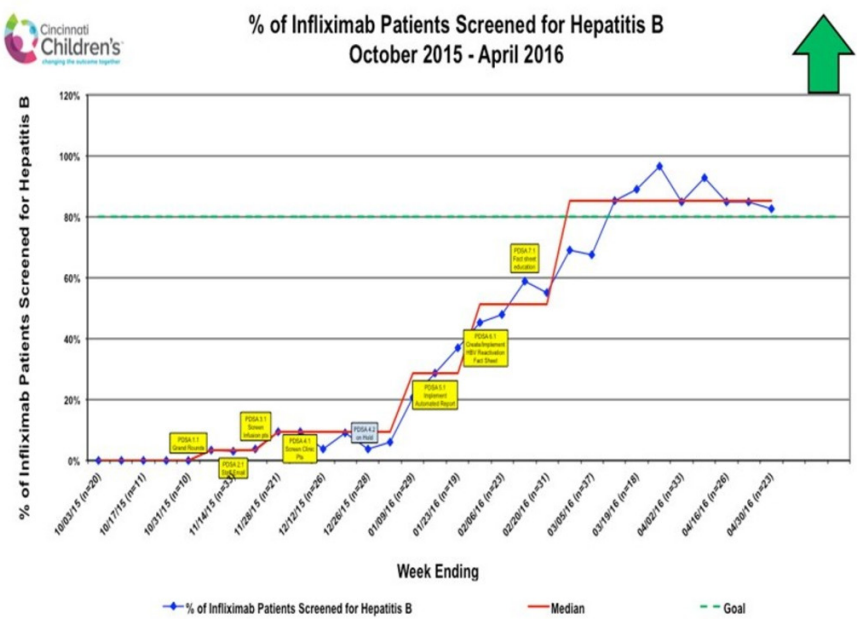

Figure 4 Run chart demonstrating the per cent of patients with inflammatory bowel disease and rheumatology on infliximab fully screened for hepatitis B from October 2015 to April 2016. PDSA, plan-do-study-act.

This PVP process ensures that these patients are properly screened for $\mathrm{HBV}$ in a timely manner, even prior to being started on immunosuppressive medications if possible, and has allowed hepatitis B screening to be incorporated with other health maintenance supervision elements. These changes were developed over a series of 6 months and are now automated and sustainable.

\section{STRATEGY}

PDSAs were performed over the course of this study to help better achieve and sustain our SMART aim.

\section{Education}

Information session within the Divisions of Pediatric Gastroenterology and Rheumatology at the CCHMC during one of their respective Grand Rounds morning educational conferences. The prediction prior to implementation of the PDSA was that buy-in for the project would be achieved by the majority of the audience members. While education was a necessary first step, this was insufficient (and has been insufficient in the past) to expect a significant sustainable change in clinical practice without other interventions. This PDSA was 'adopted' and was used in intervals during the beginning of the hepatitis B screening initiative as needed.

\section{Reminder emails/reflex entry}

Beginning with a small subset of patients (patients receiving infliximab only), labs were reviewed prior to infusion encounters and if hepatitis B titres were needed, reminder emails were sent to providers notifying them what each patient needed in order to have a complete screening performed. Physicians were asked to order labs themselves, but we offered them an 'opt-out' order entry so that if they did not notify the team otherwise, the orders would be placed on their behalf. This PDSA was effective during the early phases of the initiative, but was not sustainable due to the time and number of personnel required to complete the data collection and reminder emails each week. Ultimately the reminder emails were 'adapted' to remind only and did not include reflex order entry. This was more sustainable (and orders were still placed by primary physicians after buy-in was improved) but still needed to be adapted to a more sustainable reminder that did not include manual emails.

\section{Order sets}

In order to ensure appropriate laboratory tests being ordered, outpatient and infusion order sets were updated with appropriate labs. As physicians assumed more responsibility, this has assured that the correct labs were being ordered but did not directly affect per cent of patients screened. It was adopted as a necessary step for complete order entry.

\section{EMR build of an automated report identifying patients needing hepatitis $B$ titres}

In order to identify patients more rapidly and offset the manual work being required to identify patients, automated reports of patients in need of hepatitis B immunity screening or immunisation were developed. This allowed rapid weekly identification of patients in need of labs or immunisations. This was adopted as a necessary intervention for identification of patients, but was again believed that some 'adaptation' would be necessary prior to scaling up to include all clinic patients.

\section{Fact sheet and screening/immunisation algorithm}

After initial hepatitis B screening began, we identified a gap in provider knowledge about how and when to provide subsequent hepatitis B vaccines. In order to meet this need, a 'fact sheet', as well as 'talking points' surrounding hepatitis B vaccination and immunity, were created for providers and nurses. While this did not affect the run chart directly, provider and nurse comfort level did seem to improve and led to more independent order entry of labs and subsequent vaccines.

\section{RESULTS}

We have seen a substantial improvement in the percentage of patients receiving infliximab who were documented to have received complete hepatitis B screening, increasing from $0 \%$ to almost $90 \%$ (figure 4 ). We identified a substantial number of at-risk patients during this time with up to $71 \%$ of screened patients with IBD not showing immunity at the time of initial screening. Of these patients, $66 \%$ subsequently received a dose of either licensed single-antigen hepatitis B vaccine (Engerix-B or Recombivax HB). At this time, roughly $50 \%$ of these patients with IBD sero-converted after receiving a hepatitis $B$ vaccine. It is important to note that $\sim 34 \%$ of the patients with IBD that received a vaccine have not yet had a postvaccine anti-HBs measured; therefore, the percentage of patients with IBD that have sero-converted after receiving a hepatitis $B$ vaccine in our cohort is likely to be higher than 
$50 \%$. Further work will continue to evaluate the success of immunisation in these patients and will be expanded to patients who may not have initiated immunosuppressive medications like infliximab, which may result in reduced responsiveness to the vaccine.

\section{LESSONS AND LIMITATIONS}

One of the major limitations encountered in the system revolved around difficulty integrating knowledge of guidelines for immunisation screenings into daily clinical care. Repeated exposure with information, simplified processes and reminders, as well as simplifying order entry have all helped with buy-in and are now a part of expected and routine care within the GI and rheumatology divisions.

Another major limitation surrounded the lack of automated processes. Successful PDSAs reduced cumbersome extra steps, eliminated the need for providers or patients to recall steps on their own and allowed for automated process completion rather than manual work. At the completion of the hepatitis B improvement work, there remain a number of steps that are not automated and include automatic order entry by clinic staff (which has been beneficial for other vaccine projects in our clinics), as well as communication with primary physician regarding vaccine administration which will continue to be part of future work. While this project achieved its aim, the weekly patient identification and screenings, automated patient lists, easy-to-use order sets and immunisation algorithms have served as a starting point for other work aimed at centralising and incorporating other health maintenance improvement projects into one simple process for all patients at every planned encounter.

\section{CONCLUSIONS}

We demonstrated that by providing education and achieving consensus surrounding hepatitis B screening, automating processes and simplifying algorithms for testing and order entry, we could improve the implementation of recommended screening guidelines for hepatitis B immunity in at-risk patients receiving infliximab in paediatric IBD and rheumatic disease populations. Subsequent work is being developed using PDSAs from the hepatitis B screening process including previsit automated patient identification, and screening and vaccination algorithm. We will continue to work to integrate this work with other ongoing improvement projects in an effort to create a centralised and automated health maintenance supervision process for our clinic and infusion visits.

Acknowledgements This work was completed due to support from the $\mathrm{NIH}$ Training grant T32 DK007727.
Contributors Conception or design of the work: LAD, DMHD, MRJ, AW and TM-D. Data collection: MRJ, DMHD, AW, TM-D, KK, PM, JH, EAS, LF and EW. Data analysis and interpretation: MRJ, DMHD, LAD, PM, AW, TM-D, JH, EAS, LF and EW. Drafting the article: MRJ and DMHD. Critical revision of the article: DMHD, LAD, SAS, JH, EAS, LF, RCB and LDI. Final approval of the version to be published: DMHD, SS, LAD and MRJ.

Funding NIH T32 DK007727 PI Dr Lee A Denson; Pfizer 15448819 PI Dr Rebecca Brady.

Provenance and peer review Not commissioned; externally peer reviewed.

Open Access This is an Open Access article distributed in accordance with the Creative Commons Attribution Non Commercial (CC BY-NC 4.0) license, which permits others to distribute, remix, adapt, build upon this work non-commercially, and license their derivative works on different terms, provided the original work is properly cited and the use is non-commercial. See: http://creativecommons.org/ licenses/by-nc/4.0/

(c) Published by the BMJ Publishing Group Limited. For permission to use (where not already granted under a licence) please go to http://www.bmj.com/company/ products-services/rights-and-licensing/

\section{REFERENCES}

1. Rufo PA, Denson LA, Sylvester FA, et al. Health supervision in the management of children and adolescents with IBD: NASPGHAN recommendations. J Pediatr Gastroenterol Nutr 2012;55:93-108.

2. Weiss JE, llowite NT. Juvenile idiopathic arthritis. Rheum Dis Clin North Am 2007;33:441-70.

3. Karwowski CA, Keljo D, Szigethy E. Strategies to improve quality of life in adolescents with inflammatory bowel disease. Inflamm Bowel Dis 2009;15:1755-64.

4. Moses J, Alkhouri N, Shannon A, et al. Hepatitis B immunity and response to booster vaccination in children with inflammatory bowel disease treated with infliximab. Am J Gastroenterol 2012;107:133-8.

5. Mast EE, Margolis HS, Fiore AE, et al. A comprehensive immunization strategy to eliminate transmission of hepatitis $B$ virus infection in the United States: recommendations of the Advisory Committee on Immunization Practices (ACIP) part 1: immunization of infants, children, and adolescents. MMWR Recomm Rep 2005;54:1-31.

6. Groot N, Heijstek MW, Wulffraat NM. Vaccinations in paediatric rheumatology: an update on current developments. Curr Rheumatol Rep 2015;17:46

7. Szmuness W, Stevens CE, Harley EJ, et al. Hepatitis B vaccine: demonstration of efficacy in a controlled clinical trial in a high-risk population in the United States. N Engl J Med 1980;303:833-41.

8. Francis DP, Hadler SC, Thompson SE, et al. The prevention of hepatitis B with vaccine. Report of the centers for disease control multi-center efficacy trial among homosexual men. Ann Intern Med 1982;97:362-6.

9. Hadler SC, Francis DP, Maynard JE, et al. Long-term immunogenicity and efficacy of hepatitis B vaccine in homosexual men. $N$ Engl J Med 1986;315:209-14.

10. Jack $A D$, Hall $A J$, Maine $N$, et al. What level of hepatitis $B$ antibody is protective? J Infect Dis 1999;179:489-92.

11. Spada E, Romanò L, Tosti ME, et al. Hepatitis B immunity in teenagers vaccinated as infants: an Italian 17-year follow-up study. Clin Microbiol Infect 2014;20:0680-0686.

12. Gisbert JP, Villagrasa JR, Rodríguez-Nogueiras A, et al. Efficacy of hepatitis $B$ vaccination and revaccination and factors impacting on response in patients with inflammatory bowel disease. Am J Gastroenterol 2012;107:1460-6.

13. Millonig G, Kern M, Ludwiczek O, et al. Subfulminant hepatitis B after infliximab in Crohn's disease: need for HBV-screening? World J Gastroenterol 2006;12:974-6.

14. Ojiro K, Naganuma $M$, Ebinuma $H$, et al. Reactivation of hepatitis $B$ in a patient with Crohn's disease treated using infliximab. J Gastroenterol 2008;43:397-401.

15. Esteve M, Saro C, González-Huix F, et al. Chronic hepatitis B reactivation following infliximab therapy in Crohn's disease patients: need for primary prophylaxis. Gut 2004;53:1363-5.

16. Di Bisceglie AM, Lok AS, Martin P, et al. Recent US Food and Drug Administration warnings on hepatitis $B$ reactivation with immunesuppressing and anticancer drugs: just the tip of the iceberg? Hepatology 2015;61:703-11. 